\title{
Anhedonia in the Shadow of Chronic Social Defeat Stress, or when the Experimental Context Matters
}

\author{
N.P. Bondar, I.L. Kovalenko, D.F. Avgustinovich, D.A. Smagin and N.N. Kudryavtseva* \\ Neurogenetics of Social Behavior Sector, Institute of Cytology and Genetics SD RAS, Novosibirsk, 630090, Russia
}

\begin{abstract}
One of the core symptoms of major depression in human is anhedonia. For that reason, one of the main requirements towards experimental depression models is that they be able to demonstrate anhedonia in animals, that have been exposed to stressful events, and other behavioral changes attributable to a depression-like state. However, the results presented in the literature are contradictory: sweet solution intake, which is considered as a parameter of hedonic/anhedonic behavior in animals, responds quite differently to stressful situations in that it is either unaffected or increased or decreased. Different experimental designs used for the study of anhedonia in male mice exposed to chronic social defeat stress were tried to understand the reasons for so contradictory responses. Anhedonia appears as an abrupt reduction in sweet solution consumption in stressed animals and by failure to attain recovery after deprivation. However, it was also demonstrated that sucrose solution intake and preference strongly depend on the experimental context; that the possible critical factor may be prior acquaintance with the hedonic stimulus - or the lack whereof. Analysis of literature data and ours allowed us to conclude that the lack of a significant decrease in sweet solution intake in stressed animals is no evidence of lack of depression. Furthermore this decrease is evidence of anhedonia only provided other symptoms of depression are present. Hedonic consumable intake can be decreased over various diseases, in particular, a high level of anxiety, pathological aggression or depression.
\end{abstract}

Keywords: Ahnedonia, social defeat stress, sensory contact model, mixed anxiety/ depression state, vanillin/sucrose solution, experimental design.

\section{INTRODUCTION}

One of the core symptoms of major depression in human is anhedonia [1], which may be broadly understood as an unwillingness to do anymore whatever used to bring pleasure and satisfaction (communicate, eat, do sex, do sports) and, overall, a lack of interest in life. For that reason, one of the main requirements towards experimental depression models is that they be able to demonstrate the development of anhedonia in the animals that have been exposed to stressful events and also exhibit other behavioral changes that are indicative of a depression-like state. The normally used hedonic stimulus is an aqueous solution of sucrose or saccharine, which the animals, after some time, begin to prefer over water. It has been demonstrated that unpredictable physical stress, chronic mild stress, chronic social defeat stress, various combined stresses are able to reduce sucrose or saccharine consumption in animals [2-8]. It has been assumed that decreased sucrose consumption is an indication of anhedonia and, therefore, depression in those animals.

However, poor repeatability of the measures (decrease of sucrose consumption during exposure to chronic stress) even within a single model (in particular, a chronic mild stress model [8] and the sensitivity of sweet solution consumption to too many experimental options (animal strain; preliminary

*Address correspondence to this author at the Neurogenetics of Social Behavior Sector, Institute of Cytology and Genetics SD RAS, Pr. Ak. Lavrentjeva, 10, Novosibirsk, 630090, Russia; Tel: 7383 3301106; Fax: 7383 3331278; E-mail: natnik@bionet.nsc.ru deprivation of water or food; slight differences in experimental design; the use of different types of control animals) raised doubt as to whether decrease in sucrose consumption resulted from stress [9-12]. Therefore, it was questionable whether the animals really developed a depression-like state.

\section{ANALYSIS OF THE APPROACHES FOR STUDY OF ANHEDONIA IN STRESSED ANIMALS}

Analysis of the experimental designs presented in other works dealing with sweet solution consumption (sucrose, saccharine) by stressed animals revealed some common features: 1) stressed animals are in social isolation during the experiment $[6,7,13] ; 2)$ before exposure to stressful stimuli, animals were offered sweet water on a time-limited basis: for 2 hours [14], for 48 hours [3, 15, 16], for three days [17], one hour [13]; 3) during exposure to stressful stimuli, sweet solution consumption over various time periods, for example, one hour [3, 13] or 24 hours $[6,7]$ was measured periodically. Different authors set different intervals between measurements. In some experiments, animals were deprived of water and food for 20-21 hours [13, 15, 16], one hour [18], three hours [3] or of only water for two hours [17]. Some authors did not resort to water or food deprivation [19-22]. Under stress, sweet solution intake was either not changed $[13,15,16]$ or increased [18] or decreased [3, 7, 14, 19-28].

Many authors [9-12] argue that sucrose consumption and sucrose preference are no reliable measures in chronic stress experiments. However, analysis of literature data strongly suggests that this statement in fact originates from a correct inference made based on traditional yet erroneous data interpretation. This misinterpretation, we believe, stems from 
unawareness of the true relationship between hedonic behavior towards a preferred food (solution of sucrose or saccharin) and anhedonia, which, the researchers believe, appears as a decrease in the consumption of that food by stressed animals. A decrease in sweet water consumption is commonly interpreted as evidence of anhedonia developed due to experiencing chronic stress, and the animals that begin to drink it less than they have usually done are considered depressed. There being no decrease or it being transient, there is no depression-like state, they say.

We have demonstrated in previous studies that $\mathrm{C} 57 \mathrm{BL} / 6 \mathrm{~J}$ strain male mice exposed to or affected by chronic social defeat stress develop a mixed anxiety/depression state or anxious depression, many symptoms of which are similar to those in depressive patients [29-31]. Knowing in advance that the mice were depressed, we examined their sucrose consumption in the two-bottle free-choice paradigm, in various experimental situations [32-34] that in our opinion could account for the variability of results obtained using other depression models.

\section{CHRONIC SOCIAL DEFEAT STRESS AND ITS CONSEQUENCES}

\subsection{Mixed Anxiety/Depression State in Mice}

Validity of mixed anxiety/depression state evoked by chronic social defeat stress in male mice of the C57BL/6J strain under the sensory contact model [35, 36] was confirmed in numerous experiments. Pairs of animals were placed in cages each bisected by a perforated transparent partition allowing the animals to see, hear and smell each other, but preventing physical contact. After two days of adaptation to the housing conditions and sensory contact, testing commenced. Every day the partition was removed for 10 minutes to allow agonistic interactions to start. The superiority of one of the mice was firmly established within three test sessions. A superior mouse would be attacking, biting and chasing another, who would display only defensive behavior (sideways postures, upright postures, withdrawal, lying on the back or freezing). The mice were allowed to fight for no longer than three minutes, at which point the encounter was discontinued by pulling down the partition. After the fight, each defeated mouse was placed in an unfamiliar cage with an unfamiliar winner behind the partition. The winners remained in their original cages. The procedure yielded equal numbers of victorious mice (aggressive mice, winners) and defeated mice (submissive mice, losers).

As has been earlier shown [reviews, 29-31, 37], social defeat repeatedly experienced in agonistic interactions leads to dramatic changes in social and individual behaviors, as well as welfare. The losers developed a severe behavioral deficit: after experiencing social defeat for 20 days, they could only demonstrate a passive defense and immobile postures, whilst in the beginning they preferred active defense and withdrawal. They displayed reduced ambulation in the open-field, increased immobility time in the Porsolt's test and demonstrated no aggression in either, no matter how provoking the situation. Clear-cut anxiety was revealed in the plus-maze test and disturbed social behavior was revealed in the partition test [38]. A loss of weight, decreased plasma testosterone levels [37], decreased immune responsiveness [39], and decreased stress reactivity [29] have been demonstrated, too. Repeated treatment with the antidepressants imipramine [29] or tianeptine [37] decreased immobility in the Porsolt's test. Chronic ethanol consumption [40] and chronic treatment with ipsapirone [41] reduced anxiety, but did not prevent depression. Chronic unavoidable social stress is believed to be a pathogenic factor that leads to a mixed anxiety/depression state in mice [30]. Changes in brain serotonergic activity were detected in mice losing fights [review, 31]. The data obtained were interpreted as evidence of depression, because all the criteria proposed by McKinney and Bunney [42] were met: The etiology, response to treatment, symptomatology and brain neurochemical changes are analogous to those of human depression [30]. It has also been demonstrated using a similar experimental design that symptoms of depression would appear in response to social stress and that this state is sensitive to antidepressants $[6,43,44]$. If it were found that mice would reduce sucrose consumption in response to chronic social stress, anhedonia could be suspected.

\subsection{The control}

Since 1988 the controls both for winners and losers received under the sensory contact model were the mice that had been housed individually for five days (a shorter-term individual housing would not have eliminated the submissiveness of group-housed males, and, at longer times, social isolation would have become a factor). They were thought to be the better controls with native social and emotional responses. The rationale, correctness and advantage of this control have been explained [35, 36]. Special investigations have demonstrated that animals after five days of individual housing are less anxious, have larger exploratory and motor activity as well as higher level of communicativeness in comparison with other possible controls which could be used to chronically stressed animals - animals after long social isolation, group-housed animals etc. [45].

The mean value of sucrose solution intake by the singlehoused mice over four days beginning from the second day were used as the control consumption. It was presumed [32] that on the first day the animals are tasting sucrose solution and on the others they already prefer it. In all the experiments described below, sucrose solution intake or/and preference were higher in the controls than in any other group of animals: winners, losers, or group-housed animals at least on one measurement occasion. Thus, we can think, that sucrose solution intake by such controls reflects the hedonic preference of intact animals.

\section{EXPERIMENTAL DESIGNS FOR THE STUDY OF ANHEDONIA DEVELOPED IN MICE UNDER SOCIAL DEFEAT STRESS}

Because sucrose preference takes about two months to develop using the two-bottle free-choice paradigm [46], to boost the process, we used $1 \%$ aqueous sucrose solution supplemented with vanillin to a concentration of $0.2 \%$ [33]. Preliminary experiments demonstrated that group-housed mice given the two-bottle free-choice regimen with access to water and either vanillin/sucrose or sucrose solution would willingly drink the sweet solution. However, after the bottles containing the vanillin/sucrose solution were swapped with 
the bottles containing the sucrose solution, mice would drink significantly more vanillin/sucrose solution. Because vanillin/sucrose solution was more attractive to the mice, we chose to use it.

\subsection{Consumption of Vanillin/Sucrose Solution by Losers Long Familiar with it Before Exposure to Agonistic Interactions}

Before proceeding to the main point of the experiment, it was necessary to be sure that aqueous sucrose solution supplemented with vanillin would be liked by mice and to find out whether this solution would be preferable over water when offered under a free-choice regimen. In other words, we needed to know if the sweet solution possesses the properties of a positive reinforcer or reward.

Group-housed mice were offered to choose between water and vanillin/sucrose solution for 10 days under the two-bottle free-choice regimen. The position of each bottle was changed once a day to prevent side preference. The consumption of vanillin/sucrose solution and water by group-housed mice was expressed as a ratio to the total weight of animals in the cage.
The experiment demonstrated [32] (Fig. 1, Design 1) that mice can develop hedonic behavior towards vanillin/sucrose solution. As early as on day two following introduction to vanillin/sucrose solution, the group-housed males consumed the solution at the highest rate (about $70 \%$ of total liquid intake) and consistently preferred it over water for a total of nine days (Fig. 2). Undoubtedly, this preference is because vanillin/sucrose solution is more palatable than water. It is possible that preference developed so rapidly due to the smell of vanilla, which is attractive to mice. However, sweet solution consumption per gram total body weight of the group-housed animals was considerably decreased as compared to that by the single-housed controls, and therefore, so was the total liquid intake by group-housed animals (Table 1). This decreased vanillin/sucrose solution consumption may result from the hierarchical relationships that exist within the groups, and so not all animals have equal access to the solution, which is a source of pleasure. Furthermore, it is also possible that subordinate males, which, in laboratory mouse populations, are all but one dominant male [47], have less interest to a novel stimulus, vanillin/sucrose solution. If this is the case, then the observed decrease may be regarded as a sign of anhedonia

Table 1. Voluntary Vanillin/Sucrose Solution Preference, Solution and Water Intake, Total Liquid Intake by Single(Control) and Group-Housed Male Mice

\begin{tabular}{|l|c|c|}
\hline \multicolumn{1}{|c|}{ Parameters } & Control & Group-Housed Mice \\
\hline \hline Vanillin/sucrose solution preference, $\%$ & $74.5 \pm 6.1$ & $65.5 \pm 5.2$ \\
\hline Vanillin/sucrose solution intake per body weight, g/g & $0.167 \pm 0.013$ & $0.114 \pm 0.013^{*}$ \\
\hline Water intake per body weight, g/g & $0.051 \pm 0.012$ & $0.060 \pm 0.010$ \\
\hline Total liquid intake per body weight, g/g & $0.209 \pm 0.007$ & $0.173 \pm 0.011^{* *}$ \\
\hline$*$ - $\mathrm{p}<0.05, * *-\mathrm{p}<0.01$ in comparison with control, t-test. & & \\
\hline
\end{tabular}

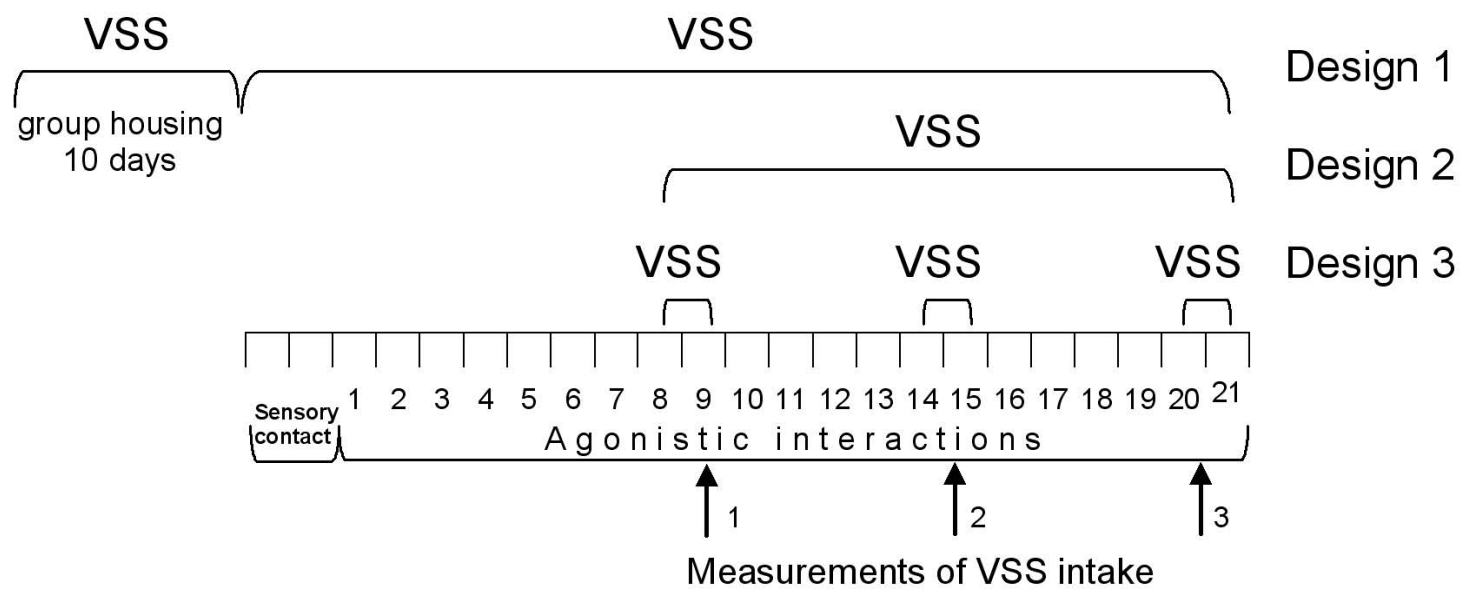

Fig. (1). Protocols of Design 1 - Vanillin/sucrose solution intake by losers long familiar with it before exposure to agonistic interactions; Design 2 - Vanillin/sucrose solution intake by losers previously unfamiliar with it; Design 3 - Vanillin/sucrose solution intake by losers under an intermittent supply scheme. Detail explanations are in text. The animals and the bottles with vanillin/sucrose solution and water were weighed once every 6 days to estimate 24 hours liquid consumption: on Day 9 (the first measurement occasion), Day 15 (the second measurement occasion), and Day 21 (the third measurement occasion). The position of each bottle was changed once a day after agonistic interactions, to prevent side preference. Daily agonistic interactions continued throughout the entire experimental period. Total liquid (water and sweet solution) intake per body weight, g/g, water intake/body weight, g/g, sweet solution intake/body weight, g/g, sweet solution preference ( $\%$ of the consumed sweet solution relative to the total amount of liquid intake) were analyzed as parameters of hedonic/anhedonic behavior. VSS - vanillin/sucrose solution supply. 


\section{Vanillin/sucrose solution $\%$}

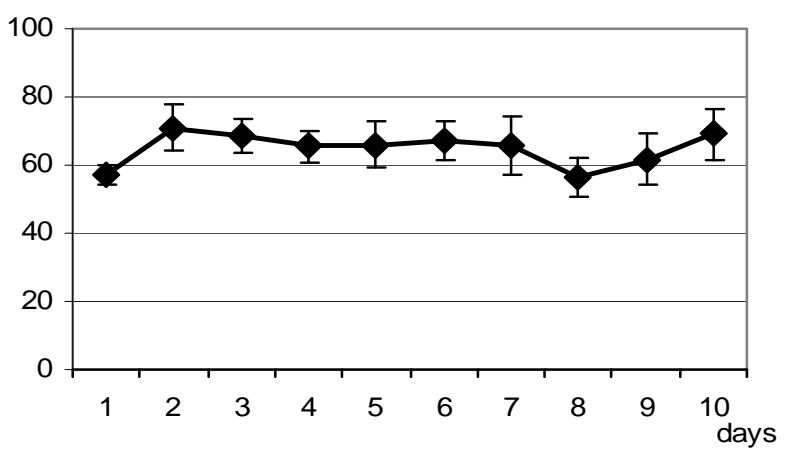

$g / 9$
Vanillin/sucrose sollution intake

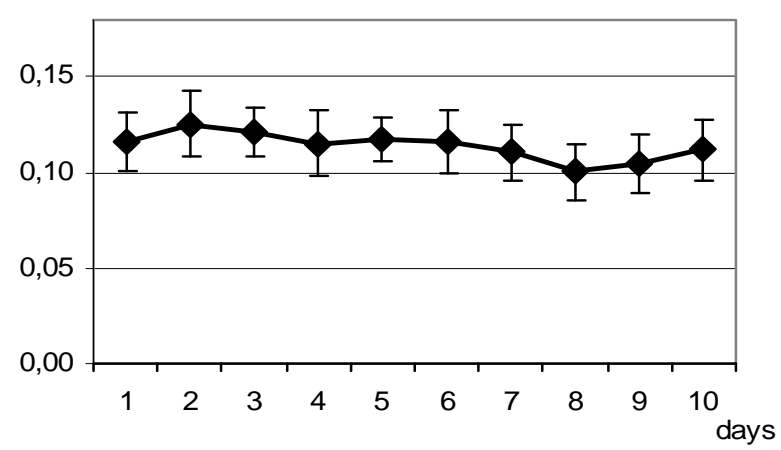

Fig. (2). Voluntary vanillin/sucrose preference $(\%)$ and solution intake $(\mathrm{g} / \mathrm{g})$ by group-housed mice. Group-housed mice were offered to choose between water and vanillin/sucrose solution for 10 days under the two-bottle free-choice regimen. The position of each bottle was changed once a day to prevent side preference [32].

developed by group-housed subordinate males.

After that period, the animals were placed in experimental cages (Fig. 1, Design 1). Losers were given for 23 days a two-bottle free-choice regimen, under which they had to choose between vanillin/sucrose solution and water. The bottles were weighed once every 6 days to estimate 24 hours liquid consumption. Daily agonistic interactions continued throughout the entire experimental period.

The mice long familiar with the taste of vanillin/sucrose solution will prefer it even while being stressed by social defeat: on all fighting days the sweet preference was about $70 \%$ (cf. $75 \%$ in the controls) relative to the total amount of liquid intake (Fig. 3). However, sweet solution consumption per body weight was significantly decreased in these mice compared to the controls, while water consumption was similar [32]. It may be possible that the sweet solution consumption rates displayed by the losers may reflect their preference which was formed during living in the groups.

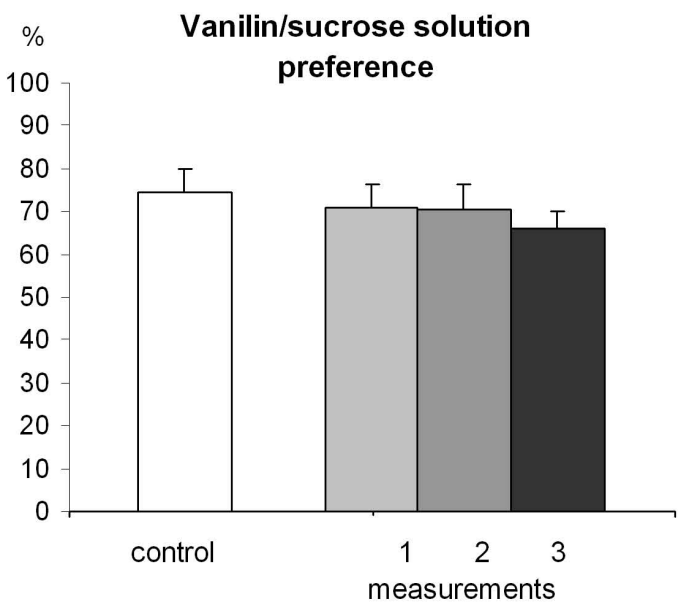

Social stress did not affect regular consumption rates. However, the losers would still drink significantly less than the controls, which may suggest that certain signs of anhedonia have appeared in the losers.

Similar data had previously been obtained on another food of choice, cheese [48]. Its has been demonstrated that given a free-choice over whether to take regular food (pellets) or cheese the losers, who had previously eaten cheese for two weeks in the groups, would prefer it over pellets ( $80 \%$ relative to the total amount of food). This may indicate that cheese is more palatable to mice than regular food, and so it could be used as a hedonic stimulus. Noteworthy, group-housed mice consumed cheese at the same (or occasionally even lower) rate for three weeks, while the losers consumed gradually more and more cheese and 18 days later they even topped the group-housed mice. It is presumable that cheese as a positive reinforcer can ease the effects of social stress by creating a positive background through palatability. Stressed animals are in more need of

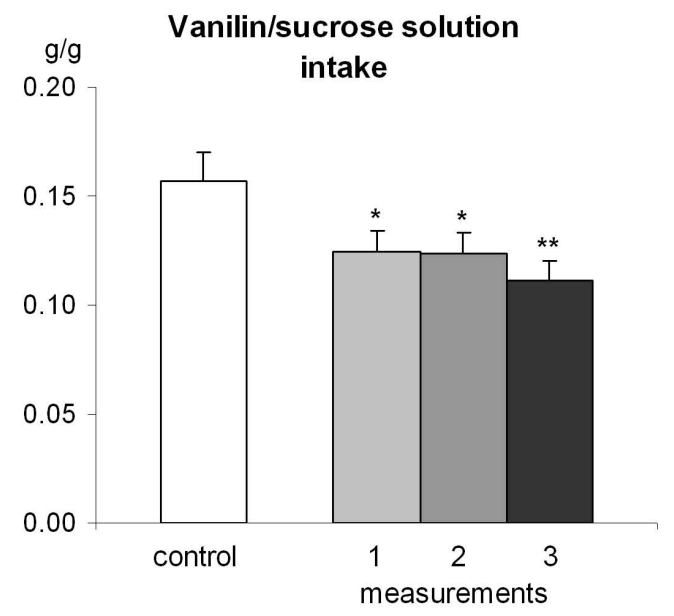

Fig. (3). Voluntary vanillin/sucrose solution preference $(\%)$ and solution intake $(\mathrm{g} / \mathrm{g})$ by male mice familiar with vanillin sucrose solution during 10 days [32]. Then male mice were placed in the condition of daily chronic social stress for 21 days under which they had to choose between vanillin/sucrose solution and water. The bottles were weighed once every 6 days to estimate 24 hours liquid consumption. 1, 2, 3 measurements on 9, 15, 21 day of agonistic interactions, respectively. $*-\mathrm{p}<0.05, * *-\mathrm{p}<0.01$, in comparison with control, t-test. 
positive reinforcement than the group-housed mice, which soon will surfeit with a mono diet (basically protein foods).

Thus, there is a common pattern of change in interest towards both consumables with similar hedonic palatability (cheese and vanillin/sucrose). Sucrose consumption preference was not decreased as compared to those in controls, and cheese consumption rates were even increased as compared to those in group-housed animals. The losers consumed much as good vanillin/sucrose solution as they had done while in the groups; however, these figures were still significantly lower than those in the controls.

\subsection{Consumption of Vanillin/Sucrose Solution by Losers Previously Unfamiliar with it}

Because the first experiment did not reveal a significant decrease of vanillin/sucrose solution preference by depressed mice previously familiar with this solution, we used another experimental design [32], in which the mice were supposed to have no prior acquaintance with it.

The protocol of this experiment is presented in Fig. (1, Design 2). After seven consecutive days of agonistic interactions, when the mice had ultimately reached opposite social statuses [35, 36], the losers were once exposed to forced vanillin/sucrose solution intake (bottles containing the sweet solution were supplied to the cages between 5.00 p.m. and 10.00 a.m). In the morning, a bottle with water was placed in each compartment to which a bottle with the sweet solution had previously been placed.

As early as on the first day and onwards, the losers took significantly less vanillin/sucrose solution than the controls and the preference was less than $25 \%$ relative to the total amount of liquid consumed (Fig. 4) [32]. Thus, when first introduced to chronically stressed animals, sweet water did not appeal to them. This decrease fits in well with the scenario of depression developed by mice exposed to chronic social stress [reviews, 29, 30, 31]: decreased vanillin/sucrose solution preference and intake by the losers may reflect the development of anhedonia and aversion to hedonic stimulus.
Possible mechanisms of this decrease are speculated on in Discussion.

\subsection{Vanillin/Sucrose Solution Intake by Losers Under an Intermittent Supply Scheme}

In many works $[3,6,7,13]$, sweet solution was given to stressed animals intermittently. In the current experiment, we, too, used intermittent supply scheme (Fig. 1, Design 3). Basically, the procedures were as described in Design 2, except for slight modifications. However, the losers were not exposed to forced vanillin/sucrose solution intake before the first measurement occasion.

When supplied intermittently, the losers drank less vanillin/sucrose solution than the controls (preference was $45 \%$ vs $75 \%$ ) (Fig. 5). However, no significant difference in consumption was found between losers and controls (the standard error was high on the losers). It may be expected that given intermittent supply with liquid in two bottles (one with sweet solution and one with water), chances of either being approached are equal and animals are drinking the whole day from the one they have approached. Fig. (6) demonstrates the individual data of vanillin/sucrose solution preference and intake after the first day of solution supply and after exposure to socially stressful conditions for 20 days. There were losers: one consuming vanillin/sucrose solution similarly with the controls and one consuming significantly less vanillin/sucrose solution than the controls. Some losers drank vanillin/sucrose solution at a lower level during first supply and then increased consumption. On the contrary there were losers which drank vanillin/sucrose solution at a higher level and then decreased consumption. There were losers which drank vanillin/sucrose solution without any changes during the entire period of social stress action. However, the means of vanillin/sucrose solution preference and intake were lower in relation to the control.

Similar results were reported by Strekalova with colleagues [7]: chronically stressed animals supplied with sweet water intermittently divided into a high and a low

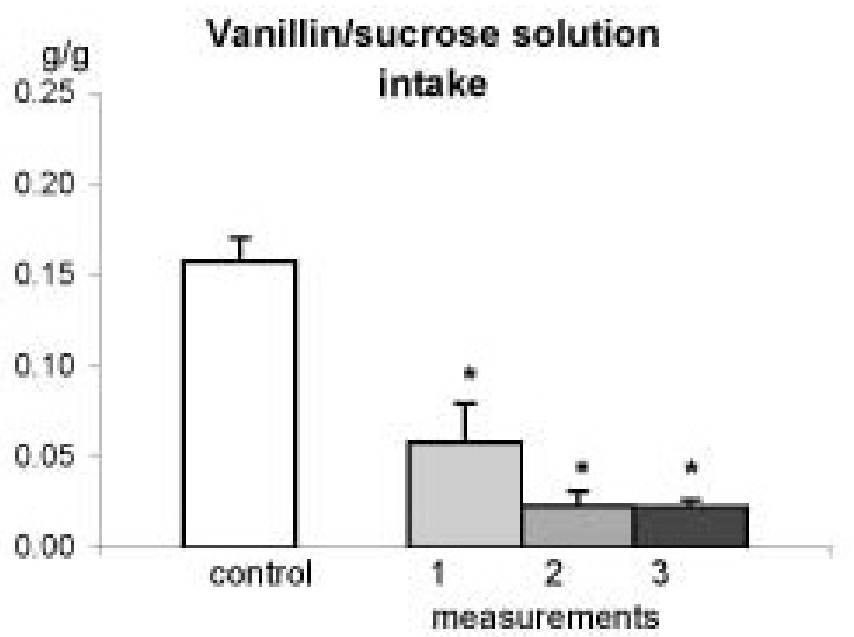

Fig. (4). Voluntary vanillin/sucrose solution preference $(\%)$ and solution intake $(\mathrm{g} / \mathrm{g})$ by male mice placed in the conditions of daily chronic social stress and non-familiar with vanillin sucrose solution [32]. After seven consecutive days of agonistic interactions, the losers were once exposed to forced vanillin/sucrose solution intake (bottles containing the sweet solution were supplied to the cages between 5.00 and 10.00 a.m). In the morning, a bottle with water was placed in each compartment to which a bottle with the sweet solution had previously been placed. 1, 2, 3 - measurements on 9, 15, 21 day of agonistic interactions, respectively. ${ }^{*}$ - p<0.001 - in comparison with control, t-test. 

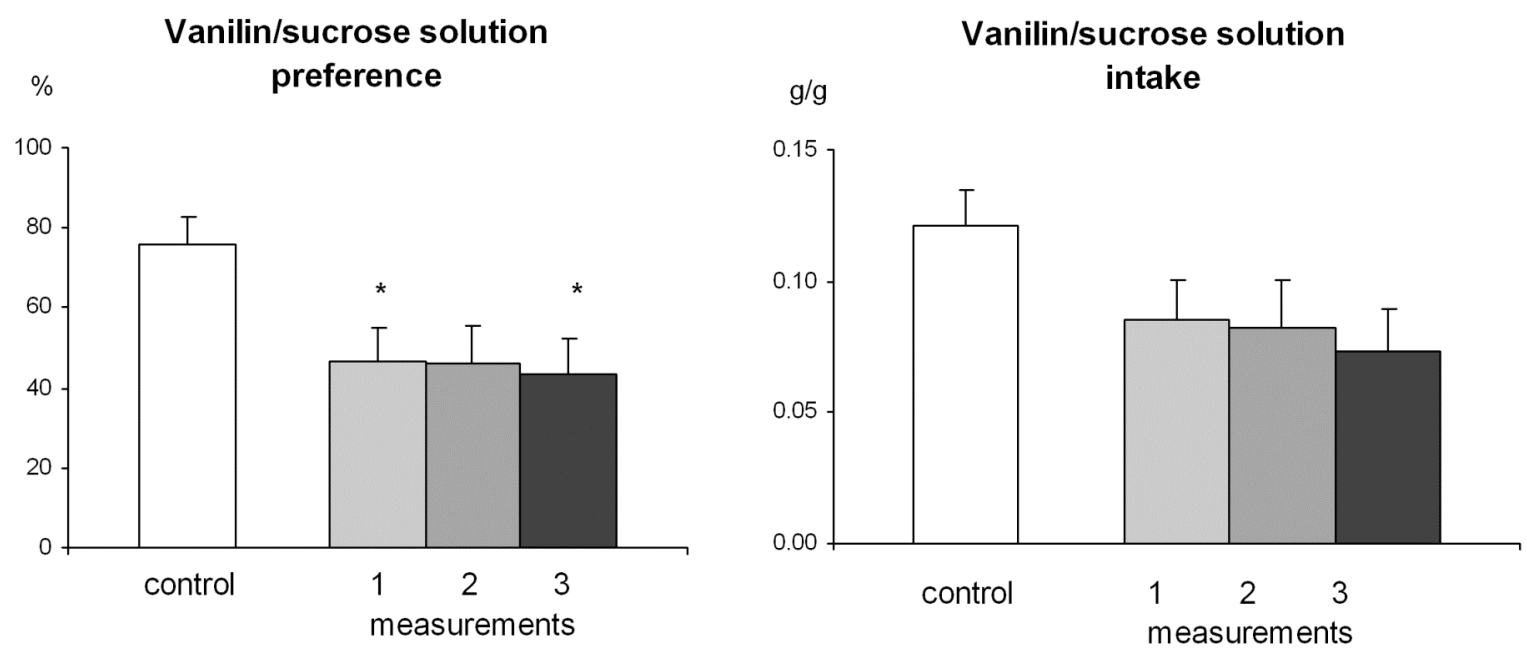

Fig. (5). Voluntary vanillin/sucrose solution preference $(\%)$ and intake $(\mathrm{g} / \mathrm{g})$ by losers under an intermittent supply scheme. The losers received vanillin/sucrose solution for six days beginning from Day 7 of agonistic interactions under a two-bottle free-choice regimen. Vanillin/sucrose solution and water intake was measured 24 hours after introduction. 1, 2, 3 - measurements on 9, 15, 21 day of agonistic interactions, respectively. $*$ - $<<0.05$ - in comparison with control, t-test.
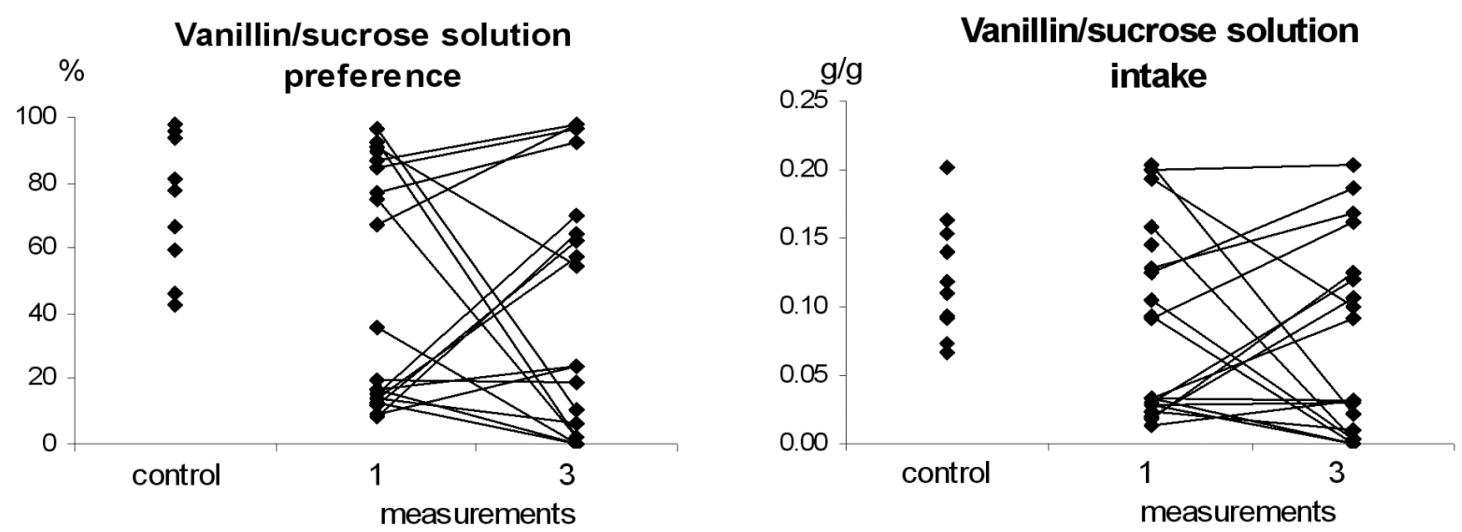

Fig. (6). Individual data of vanillin/sucrose solution preference $(\%)$ and intake $(\mathrm{g} / \mathrm{g})$ in the control and losers after the first day of solution supply and after exposure to socially stressful conditions for 21 days.

intake faction. It appears as though the changes in consumption observed in chronically stressed animals supplied with sweet solution intermittently are difficult for interpretation, because the animals had no prior acquaintance with that solution and, therefore, they cannot have developed hedonic behavior towards this drink.

\subsection{Vanillin/Sucrose Solution Intake and Deprivation Effect in Winners and Losers}

There were indications that hedonic processes may be disturbed in the animals who consistently win daily fights or the winners [33, 34, 49]. This experiment aimed to

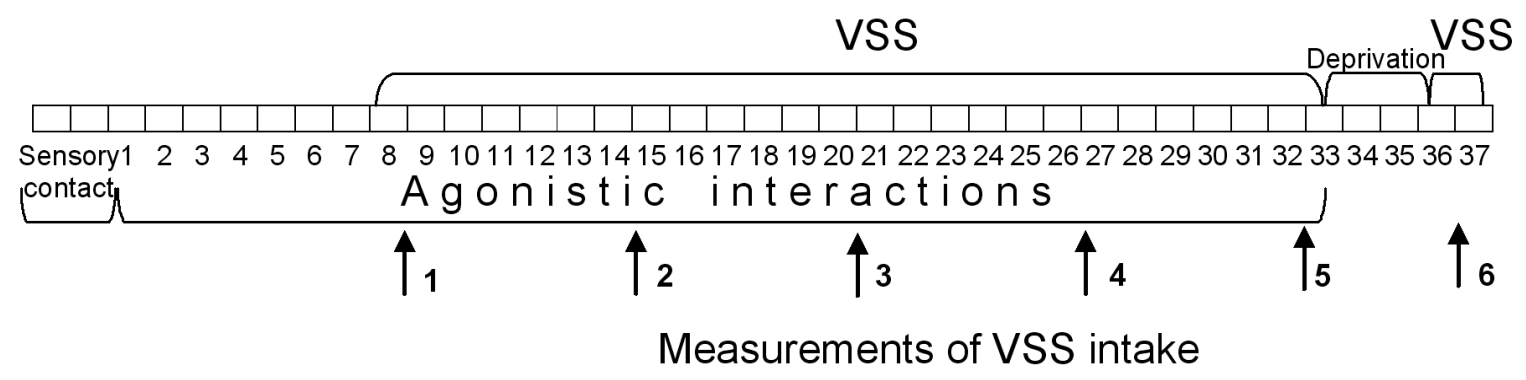

Fig. (7). Protocols of Experimental design - Vanillin/sucrose solution intake and deprivation effect in winners and losers. After seven consecutive days of agonistic interactions a two-bottle free-choice regimen was given for 25 days. Daily agonistic interactions continued throughout the entire experimental period. 1, 2, 3, 4, 5 - measurements on 9, 15, 21, 27, 33 day of fighting, respectively. Then the animals were deprived of drinking the sweet solution for three days. During the deprivation period, no agonistic interactions were allowed. After this period, the two-bottle free-choice regimen was given again, to estimate the daily sweet consumption (measurements 6). VSS vanillin/sucrose solution supply. 
Vanilin/sucrose solution preference, $\%$

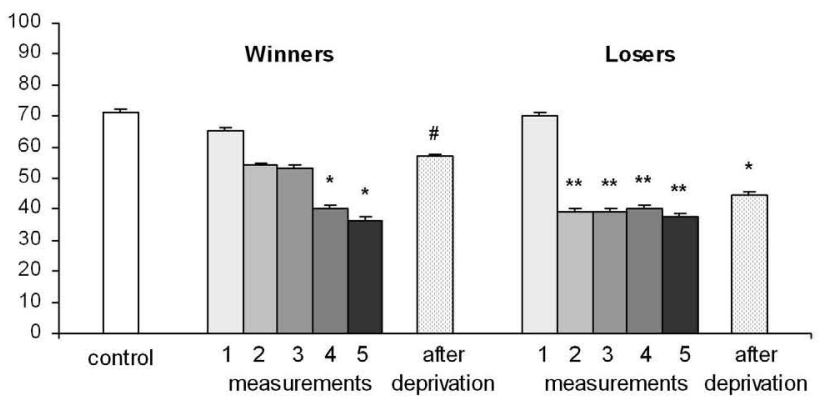

Water intake, $g / g$

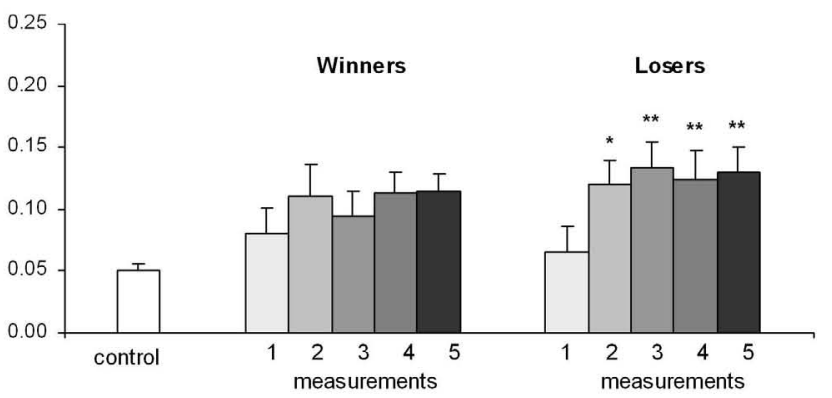

Vanilin/sucrose solution intake,g/g

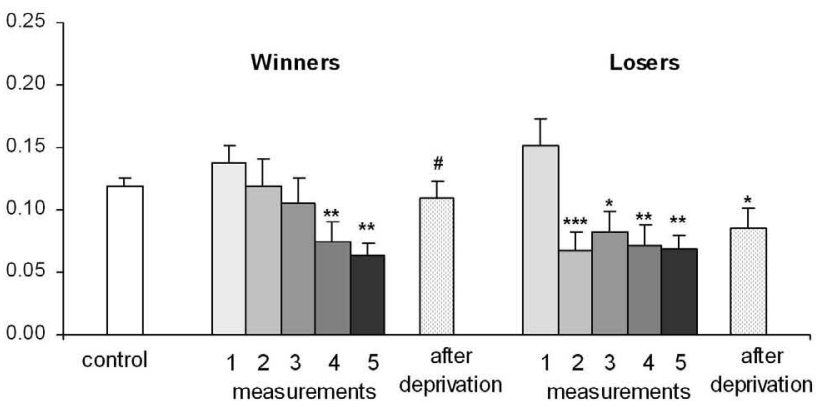

Total liquid intake, $\mathbf{g} / \mathbf{g}$

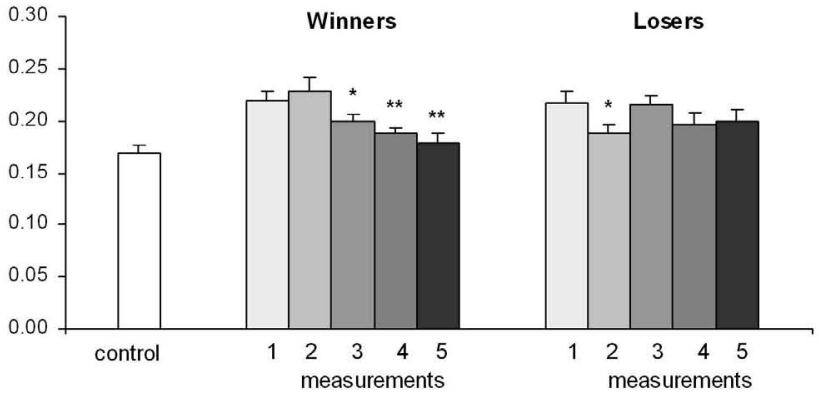

Fig. (8). Voluntary vanillin/sucrose solution preference $(\%)$, solution intake $(\mathrm{g} / \mathrm{g})$, water $(\mathrm{g} / \mathrm{g})$ and total liquid (water and sweet solution) intake $(\mathrm{g} / \mathrm{g})$ by winners and losers during fighting days [34]. $* \mathrm{p}<0.05 ; * *-\mathrm{p}<0.01$, *** $-\mathrm{p}<0.001 v$ s first-measurement figure, \# - p $<$ $0.05, v s$ fifth-measurement figure, paired t-test.

compare the amount of influence of positive and negative fighting experience in male mice on their voluntary consumption of a vanillin/sucrose solution.

The protocol of the experiment is presented in Fig. (7) [34]. A two-bottle free-choice regimen was given for 25 days. Then the animals were deprived of drinking the sweet solution for three days, because a deprivation for three days is sufficient to significantly increase post-deprivation saccharin consumption in animals [50]. During the deprivation period, no agonistic interactions were allowed. After this period, the two-bottle free-choice regimen was given again, to estimate the daily sweet consumption.

On the first day of exposure to the sweet solution, which was on Day 9 of agonistic interactions, winners and losers preferred sweet water ( $70 \%$ of the total liquid intake) similarly as the control mice did. Six days later and onwards, in the losers, sweet solution preference and intake was decreased significantly on all measurement occasions (Fig. 8). In the winners, these parameters were significantly decreased on the fourth and fifth measurement occasions, which was two weeks after the first exposure to the vanillin/sucrose solution. Thus, vanillin/sucrose solution consumption was decreased in both the victorious and defeated mice.

The finding that vanillin/sucrose solution consumption was decreased in the winners was totally unexpected. It is possible that decreased sweet solution consumption in both winners and losers might be the consequence of common processes associated with social confrontations, for example, enhanced anxiety, which is exhibited by animals with opposite social statuses [38, 51], or stress from agonistic interactions [52].

However, a careful analysis of the patterns of total liquid consumption and water intake by the winners and losers (Fig. 8) suggests that a different interpretation is possible. In the losers, total liquid intake did not change throughout the experiment. However, from the second measurement occasion on, sweet solution intake was abruptly decreased and water intake was increased, as the animals began to develop depression. The depressed losers preferred water to the sweet solution. In the victorious mice, water consumption did not change over the entire experimental period. This implies that the decreased total liquid intake observed in the winners is due to the decreased sweet solution intake. Two explanations are possible here. One is that the winners at some point reached the satiety of a novel, even though palatable liquid and finally lost interest in it. Another is that one source of positive reinforcement (vanillin/sucrose) was replaced by another, that is, the experience of being aggressive and victorious. The latter is a possibility because many [53-55] have reported that aggression is rewarding in laboratory rodents and in humans and any positive reinforcement may trigger a tendency to behave aggressively [54, 56].

The observation of the opposite effects of deprivation on sweet solution intake by winners and losers confirms the involvement of different motivational components of hedonic behavior towards this particular reinforcer. After deprivation for three days without social confrontations, sweet solution intake in the winners was recovered to the 
first-measurement level, which was similar to the control figure. In the losers, decreased vanillin/sucrose solution intake and preference did not change significantly after the deprivation.

\section{GENERAL DISCUSSION}

Analysis of our and literature data allows to suggest that the lack of tangible change in sweet solution consumption by animals in stressful conditions shown in other works may simply result from how comparison was done, i.e. what was compared with what and how. In most works, change is acknowledged when there is as a difference between sweet solution consumption by the same animals before and during exposure to stressful stimuli. In ours, we have always been comparing consumption by animals exposed to social stress with consumption by control animals, formerly grouphoused males who were single-housed for five days before used as controls. The correctness and adequacy of using such mice as the control in relation to the sensory contact model was explained elsewhere $[35,36]$ and experimentally confirmed [45]. With reliance upon these controls in the current experiments, we assumed that vanillin/sucrose solution intake by single-housed animals tells us about the gustatory and olfactory preferences typical of intact, unaffected animals with native social and emotional responses. Noteworthy, in whatever experimental settings we have placed the mice, vanillin/sucrose solution intake or/and preference has at all times been higher in the control than other experimental groups (winners and losers with shorter or longer fighting outcome history, group-housed mice etc). It may well be that the use of these particular controls allowed us to come across successful methodical tricks that the traditional experimental designs are missing. Additionally, supplementation of vanillin, which has an attractive smell for mice, to the sucrose solution was found to be a successful trick, which allowed the time required for sweet solution preference to develop to be kept to a minimum: as early as on day two following introduction the control mice had the highest preference (about $70 \%$ of total liquid intake) and this figure would stay as high for quite a long time onwards (at least 30 days under single housing).

Supportive to our understanding and our interpretation of the results are our many years worth of research, which demonstrated that social defeat repeatedly experienced in agonistic interactions leads to dramatic changes in social and individual behaviors as well as welfare. The etiology, response to treatment, symptomatology and brain neurochemical changes are analogous to those of human depression [30, 31]. And that is why the lack of decreased vanillin/sucrose solution preference by mice exposed to stressful stimuli in the first experiment just made us want to seek such experimental designs that could convincingly identify disturbances in hedonic behavior in depressed mice.

We have demonstrated a strong influence of experimental context on sweet solution intake by the losers. In the first experiment, the mice were familiar with the vanillin/sucrose solution and had a nearly $65-70 \%$ sweet solution preference when experiencing defeat stress. Thus, exposed to social stress, the mice still prefer vanillin/sucrose solution. Something similar is true of another food, cheese, that the mice eat more willingly than standard pellets [48].
Moreover, when stressed, mice would even eat some more cheese, which is something that the group-housed mice long with an option to choose between pellets and cheese would not do. We came to the conclusion [32] that if hedonic consumables become the regular and favored food, chronic stress does not decrease but even increase its consumption.

In the second and fourth experiments, this solution was given to defeat-stressed animals previously unfamiliar with it. After 5-10 days onward, there was a decrease in vanillin/sucrose solution preference by these animals as compared to the controls (40-10\% of total liquid intake). It appears as though, unlike intact animals, the losers do not want vanillin/sucrose solution - they want water. The pattern of decreased sweet solution intake in response to chronic social defeat stress superbly correlates with the development of depression in the losers as had previously been shown [29-31]. This implies that depression that we evoked using our model definitely includes its core symptom, anhedonia. Admittedly, decreased sweet solution intake had been observed in the losers before they demonstrated full-blown depression state.

As has previously been demonstrated, living in danger (which is in the presence of an aggressive partner behind the partition of the experimental cage) causes anxiety and fear in the losers. As early as on Day 3 of agonistic confrontations, the partition test revealed a decrease in communicative behavior, which was interpreted as enhanced anxiety [31]. After 10 daily fighting sessions, anxiety was even more enhanced and was sensed by the plus-maze. After 20 daily fighting sessions, anxiety became overwhelming and was sensed by all the behavioral tests performed. That is why we were speaking of a mixed anxiety/depression state in stressed mice. Thus it is possible that decreased vanillin/sucrose solution intake in mice may equally be due to enhanced anxiety or depression.

Importantly, the cessation of agonistic interactions and placement of depressed animals in the in comfortable housing conditions (for example, sharing a cage with a female) did not help [57]. The plus-maze, open-field and Porsolt's test demonstrated that a comfortable housing of depressed losers for two weeks did not reduce their anxiety or depression. As our experiments demonstrated, decreased vanillin/sucrose solution intake and preference by the losers did not change significantly after the deprivation period either. This implies that the deprivation effect phenomenon, which appears as an increased free-choice consumption of drugs, alcohol, or sweet solution [51, 58-61] does not work in the depressed mice nor is interest to the source of pleasure restored after deprivation.

A question arises as to the mechanisms underlying refusal by stressed males previously unfamiliar with the taste of sweet water to take vanillin/sucrose solution. It has been demonstrated that the senses of smell and taste in depressive patients may be affected, but later restored after treatment with antidepressant drugs [62, 63]. Consequently, the fact that the mice at certain point began to consume very little vanillin/sucrose solution may suggest decreased sensitivity to the smell of vanillin or the taste of sucrose in the solution. However, if it had been for decreased sensitivity, the mice would have been drinking as good sweet solution as water. A very low consumption of sweet solution makes us suspect 
escaping from an unfamiliar food with a strange smell and taste. This could equally be evidence of anhedonia or fear of novel stimuli or lack of interest to them or indifference, which depressed mice were noted for in our previous experimental situations [30, 48]. It appears as if the animals developed aversion to this stimulus.

At the same time it is known that major depression in human patients with may not necessarily involve a reduced sense of taste or smell [64-66]. Therefore, preference shifts may not necessarily occur in such patients. Our experiments lend additional support to this logic: interest to vanillin/sucrose solution by depressed animals may not necessarily be waning (for example, it does not happen if animals have long been familiar with its taste). Moreover, some stresses can make animals and humans increase preferred food consumption [18, 67]. For that reason, the sole lack of decrease in vanillin/sucrose solution intake by animals exposed to stress should not be seen as sufficient evidence of a lack of the depression-like state. Probably it is not so much decrease in preferred sweet consumption that is the main evidence of anhedonia as the lack of the postdeprivation effect that we have experimentally demonstrated [33].

In addition, not only depression but a whole list of neurological diseases [68], Alzheimer's disease [69], and multiple sclerosis [70] causes taste and smell disturbances in human patients. In our experiments, we could observe a decrease in vanillin/sucrose solution intake by aggressive males who had won many fights and whose behavior was utterly devoid of any sign of depression. Instead, the winners demonstrated enhanced aggression and impulsivity, increased motor activity, hostility, malignancy etc. [review, 49]. Therefore, this observation challenges the generally accepted paradigm that anhedonia measured by reduced sucrose intake in rats and mice is a key symptom of depression - at least in animal models.

It seems to be most reasonable to come to an understanding of when sweet solution intake decrease can be regarded as evidence of animal anhedonia and when it cannot. To begin with, anhedonia is the coined term for the core symptom of major depression in humans. Decreased sweet intake may be interpreted as anhedonia only in depressed animals that exhibit many more signs of depression developed due to chronic social stress. Therefore, we may not loosely apply this term to the winners who have decreased sweet solution intake. It has been shown that, in the winners, aggressive motivation becomes dominated in various different experimental situations [49]: experienced winners may vigorously attack a much huger male or a fully submissive mice or even bite the experimenter's hand just because it was put in the cage. No intact male would ever do that when first confronted with a conspecific. At the same time, winners exposed to a receptive female showed a significantly weaker behavioral response [71] and their testosterone level was not increased. When first confronted with a female, they can even attack her. Recently, pathological aggression towards females in similar settings has also been demonstrated [72]. Considering both decreased vanillin/sucrose solution intake and disturbed sexual behavior toward receptive females, it may be concluded that, in the winners, the hedonic reaction to rewards such as food and sex is decreased, while aggression is increased. It may be presumed that, concerning the winners, the substitution of hedonic food motivation for aggression takes place. After deprivation, which was a time when the winners were kept away from the arena and were not getting winning pleasure, vanillin/sucrose solution intake will increase. It can therefore be claimed that decrease in vanillin/sucrose solution intake may be the effect of pathological aggression that victorious mice develop as they win more and more fights [49].

Another hypothesis, which is easy to come to, is that shifts in hedonic preferences may occur in healthy individuals due to habituation to a source of pleasure or the substitution of one food stimulus for another. It is easy to picture a situation when a new dominant motivation abrogates all the others that were there before. And, yes, any individual possesses their own selection of positive emotional stimuli, which depend on the past history, habits, inherited neurophysiological features and whatever else that may have nothing to do with foods.

\section{CONCLUDING REMARKS}

It has been earlier shown that C57BL/6J strain male mice exposed to or affected by chronic social defeat stress develop a mixed anxiety/depression state, many symptoms of which are similar to those in patients with depression, in particular, anhedonia towards a positive reinforcer which was $1 \%$ aqueous solution of sucrose supplemented with $0.2 \%$ vanillin. Anhedonia appears as an abrupt reduction in sweet solution consumption and by failure to attain recovery after deprivation. However, it was also demonstrated that vanillin/sucrose solution intake and preference under the two-bottle free-choice regimen strongly depend on the experimental context; that the possible critical factor may be prior acquaintance with the hedonic stimulus - or the lack whereof. Analysis of our original and literature data strongly suggests that the lack of a significant decrease in sweet solution intake in stressed animals is no evidence of lack of depression. Furthermore this decrease is evidence of anhedonia only provided other symptoms of depression are present. Hedonic consumable intake can be decreased over various conditions or diseases, in particular, a high level of anxiety or pathological aggression Moreover hedonic consumable intake can be decreased over habituation to a particular source of pleasure or the substitution of the food motivation by another motivation, which brings more pleasure than the sweet solution.

\section{ACKNOWLEDGEMENTS}

This work was supported by grant No. 07 04-00014 from the Russian Foundation for Basic Research. We are thankful to V.Filonenko for the title and a thorough revision of this manuscript.

\section{REFERENCES}

[1] DSM-IV. Diagnostic and statistical manual of mental disorder (DSM-IV), fourth ed. APA: Washington DC 1994.

[2] Katz RJ. Animal model of depression: pharmacological sensitivity of a hedonic deficit. Pharmacol Biochem Behav 1982; 16(6): 9658

[3] Monleon S, D'Aquila P, Parra A, Simon VM, Brain PF, Willner P. Attenuation of sucrose consumption in mice by chronic mild stress and its restoration by imipramine. Psychopharmacology (Berl) 1995; 117(4): 453-7. 
[4] Moreau JL. Simulating the anhedonia symptom of depression in animals. Dialog Clin Neurosci 2002; 4(4): 351-60.

[5] Pothion S, Bizot JC, Trovero F, Belzung C. Strain differences in sucrose preference and in the consequences of unpredictable chronic mild stress. Behav Brain Res 2004; 155(1): 135-46.

[6] Rygula R, Abumaria N, Flugge G, Fuchs E, Ruther E, HavemannReinecke U. Anhedonia and motivational deficits in rats: impact of chronic social stress. Behav Brain Res 2005;162(1): 127-34.

[7] Strekalova T, Spanagel R, Bartsch D, Henn FA, Gass P. Stressinduced anhedonia in mice is associated with deficits in forced swimming and exploration. Neuropsychopharmacology 2004; 29(11): 2007-17.

[8] Willner P. Validity, reliability and utility of the chronic mild stress model of depression: a 10-year review and evaluation. Psychopharmacology (Berl) 1997; 134(4): 319-29.

[9] Hagan JJ, Hatcher JP. Revised CMS model. Psychopharmacology (Berl) 1997; 134(4): 354-6.

[10] Harris RBS, Zhou J, Youngblood BD, Smagin GN, Ryan D.H. Failure to change exploration or saccharin preference in rats exposed to chronic mild stress. Physiol Behav 1997; 63(1): 91-100.

[11] Reid I, Forbes N, Stewart C, Matthews K. Chronic mild stress and depressive disorder: a useful new model? Psychopharmacology (Berl) 1997; 134(4): 365-7.

[12] Weiss JM. Does decreased sucrose intake indicate loss of preference in CMS model? Psychopharmacology (Berl) 1997; 134(4) :368-70.

[13] Nielsen CK, Arnt J, Sanchez C. Intracranial self-stimulation and sucrose intake differ as hedonic measures following chronic mild stress: interstrain and interindividual differences. Behav Brain Res 2000; 107(1-2): 21-33.

[14] Strekalova T, Gorenkova N, Schunk E, Dolgov O, Bartsch D. Selective effects of citalopram in a mouse model of stress-induced anhedonia with a control for chronic stress. Behav Pharmacol 2006; 17(3): 271-87.

[15] Forbes NF, Stewart CA, Matthews K, Reid IC. Chronic mild stress and sucrose consumption: validity as a model of depression. Physiol Behav 1996; 60(6): 1481-4.

[16] Matthews K, Forbes N, Reid IC. Sucrose consumption as an hedonic measure following chronic unpredictable mild stress. Physiol Behav 1995; 57(2): 241-8.

[17] Green TA, Alibhai IN, Unterberg S, et al. Induction of activating transcription factors (ATFs) ATF2, ATF3, and ATF4 in the nucleus accumbens and their regulation of emotional behavior. J Neurosci 2008; 28(9): 2025-32.

[18] Pijlman FT, Wolterink G, Van Ree JM. Physical and emotional stress have differential effects on preference for saccharine and open field behaviour in rats. Behav Brain Res 2003; 139: 131-8.

[19] Bertrand E, Smadja C, Mauborgne A, Roques BR, Dauge V. Social interaction increases the extracellular levels of met-enkephalin in the nucleus accumbens of control but not of chronic mild stressed rats. Neuroscience 1997; 80: 17-21.

[20] Muscat R, Willner P. Suppression of sucrose drinking by chronic mild unpredictable stress: a methodological analysis. Neurosci Biobehav Rev 1992;16: 507-17.

[21] Cheeta S, Broekkamp C, Willner P. Stereospecific reversal of stress-induced anhedonia by mianserin and its (+)-enantiomer. Psychopharmacology (Berl) 1994; 116(4): 523-8.

[22] Griffiths J, Shanks M, Anisman H. Strain-specific alterations in consumption of a palatable diet following repeated stressor exposure. Pharmacol Biochem Behav 1992; 42: 219-27.

[23] Ayensu WK, Pucilowski O, Mason GA, Overstreet DH, Rezvani AH, Janowsky DS. Effects of chronic mild stress on serum complement activity, saccharin preference, and corticosterone levels in Flinders lines of rats. Physiol Behav 1995; 57(1): 165-9.

[24] D'Aquila PS, Newton J, Willner P. Diurnal variation in the effect of chronic mild stress on sucrose intake and preference. Physiol Behav 1997; 62(2): 421-6.

[25] Muscat R, Towell A, Willner P. Changes in dopamine autoreceptor sensitivity in an animal model of depression. Psychopharmacology (Berl) 1988; 94(4): 545-50.

[26] Pucilowski O, Overstreet DH, Rezvani AH, Janowsky DS. Chronic mild-induced anhedonia: greater affect in a genetic rat model of depression. Physiol Behav 1993; 54: 1215-20.

[27] Sampson D, Willner P, Muscat R. Reversal of antidepressant action by dopamine antagonists in an animal model of depression. Psychopharmacology 1991; 104: 491-5.
[28] Willner P, Towell A, Sampson D, Sophokleous S, Muscat R. Reduction of sucrose preference by chronic unpredictable mild stress, and its restoration by a tricyclic antidepressant. Psychopharmacology (Berl) 1987; 93(3): 358-64.

[29] Kudryavtseva NN, Bakshtanovskaya IV, Koryakina LA. Social model of depression in mice of C57BL/6J strain. Pharmacol Biochem Behav 1991; 38(2): 315-20.

[30] Kudryavtseva NN, Avgustinovich DF. Behavioral and physiological markers of experimental depression induced by social conflicts (DISC). Aggress Behav 1998; 24: 271-86.

[31] Avgustinovich DF, Alekseyenko OV, Bakshtanovskaya IV, et al. Dynamic changes of brain serotonergic and dopaminergic activities during development of anxious depression: experimental study. Adv Physiol Sci (Usp Fiziol Nauk) 2004; 35(4): 19-40 (Russian).

[32] Bondar NP, Kovalenko IL, Avgustinovich DF, Kudryavtseva NN. Influence of experimental context on the development of anhedonia in male mice imposed to chronic social stress. I P Pavlov J Higher Nerv Activ (Zh Vyssh Nerv Deiat Im I P Pavlova) 2008; 58(2): 238-46 (Russian).

[33] Kudryavtseva NN, Bondar NP, Kovalenko IL, Avgustinovich DF. Decrease of vanillin sucrose intake by victorious and defeated mice: development of anhedonia? Available from Nature Preceding 2007 (<http://hdl.nature.com/10101/npre.2007.988.1>hdl:10101/npre.2007.9 $88.1 \mathrm{http} / /$ precedings.nature.com/documents/988/version/1)

[34] Kudryavtseva NN, Bondar NP, Kovalenko IL. Effect of positive and negative social experiences on sucrose solution intake in male mice. I P Pavlov J Higher Nerv Activ 2009; 59(2): 192-8.

[35] Kudryavtseva NN. The sensory contact model for the study of aggressive and submissive behaviors in male mice. Aggress Behav 1991; 17(5): 285-91.

[36] Kudryavtseva NN. Agonistic behavior: a model, experimental studies, and perspectives. Neurosci Behav Physiol 2000; 30(3): 293-305.

[37] Kudryavtseva NN, Bakshtanovskaya IV, Avgustinovich DF, et al. Social defeats, depression and anxiety: an experimental model. Novosibirsk: Institute of Cytology and Genetics SD RAS 1995; P. 48.

[38] Avgustinovich DF, Alekseyenko OV, Kudryavtseva NN. Comparative analysis of anxiety-like behavior in partition and plusmaze tests after agonistic interactions in mice. Physiol Behav 1997; 61(1): 37-43

[39] Devoino L, Alperina E, Kudryavtseva N, Popova N. Immune responses in male mice with aggressive and submissive behavior patterns: strain differences. Brain Behav Immun 1993; 7(1): 91-6.

[40] Kudryavtseva NN, Madorskaya IA, Bakshtanovskaya IV. Social success and voluntary ethanol consumption in mice of C57BL/6 J and CBA/Lac strains. Physiol Behav 1991; 50(1): 143-6.

[41] Avgustinovich DF, Alekseyenko OV, Koryakina LA. Chronic treatment with ipsapirone affects anxiety, but not depression in mice. Eur Neuropsychopharmacol 1999; 9(5): 301.

[42] McKinney WTJ, Bunney WEJ. Animal model of depression. I. Review of evidence: implications for research. Arch Gen Psychiatry 1969; 21: 240-8.

[43] Keeney AJ, Hogg S. Behavioural consequences of repeated social defeat in the mouse: preliminary evaluation of a potential animal model of depression. Behav Pharmacol 1999; 8: 753-64.

[44] Berton O, McClung CA, Dileone, et al. Essential role of BDNF in the mesolimbic dopamine pathway in social defeat stress. Science 2006; 311 (5762): 864-8.

[45] Avgustinovich DF, Kovalenko IL, Bondar' NP. Choice of "control" in experimental researches of animal social interactions in mice. Ross Fiziol Zh Im I M Sechenova 2005; 91(12): 1454-68. Russian

[46] Belozertseva IV, Sukhotina IA, Vossen JM, Bespalov AY. Facilitation of aggressive and sexual behaviors by saccharin deprivation in rats. Physiol Behav 2004; 80(4): 531-9.

[47] Mackintosh JH. Territory formation by laboratory mice. Anim Behav 1970; 18: 177-83.

[48] Kudriavtseva NN, Avgustinovich DF, Kovalenko IL, Bondar' NP. Development of anhedonia under negative experience of social confrontations in male mice. Ross Fiziol $\mathrm{Zh} \mathrm{Im} \mathrm{I} \mathrm{M} \mathrm{Sechenova}$ 2006; 92(3): 351-61 (Russian).

[49] Kudryavtseva NN. Psychopathology of repeated aggression: a neurobiological aspect. In: Morgan JP, Ed. Perspectives on the Psychology of Aggression. New York: NOVA Science Publishers, Inc 2006; pp. 35-64. 
[50] Neznanova ON, Zvartau EE, Bespalov AY. Behavioral analysis of the saccharin deprivation effect in rats. Behav Neurosci 2002; 116(5): 747-56

[51] Kudryavtseva NN, Bondar NP, Avgustinovich DF. Association between experience of aggression and anxiety in male mice. Behav Brain Res 2002; 133(1): 83-93.

[52] Osadchuk AV. Microevolutionary aspects of adrenocortical and gonadal functions. In Naumenko EV, Popova NK, Eds. Ontogenetic and genetic-evolutionary aspects of neuroendocrine stress regulation. Novosibirsk: Nauka 1991; PP. 160-70.

[53] Fish EW, De Bold JF, Miczek KA. Aggressive behavior as a reinforcer in mice: activation by allopregnanolone. Psychopharmacology (Berl) 2002; 163(3-4): 459-66.

[54] Moyer KE. Violence and aggression. N.Y.: Paragon House. 1987.

[55] Scott JP. Theoretical issues concerning the origin and causes of fighting. In: Eleftheriou BE, Scott JP, Eds. The physiology of aggression and defeat. New-York: Plenum1971; pp. 11-42.

[56] Baron RA, Richardson D. Human aggression. New York: Plenum Press 1994.

[57] Avgustinovich DF, Kovalenko IL, Kudryavtseva NN. A model of anxious depression: persistence of behavioral pathology. Neurosci Behav Physiol 2005; 35(9): 917-24.

[58] Holter SM, Engelmann M, Kirschke C, Liebsch G, Landgraf R, Spanagel R. Long-term ethanol self-administration with repeated ethanol deprivation episodes changes ethanol drinking pattern and increases anxiety-related behaviour during ethanol deprivation in rats. Behav Pharmacol 1998; 9(1): 41-8.

[59] Sinclair JD, Li TK. Long and short alcohol deprivation: effects on AA and P alcohol-preferring rats. Alcohol 1989; 6(6): 505-9.

[60] Spanagel R, Holter SM. Long-term alcohol self-administration with repeated alcohol deprivation phases: an animal model of alcoholism? Alcohol Alcohol 1999; 34(2): 231-43.

[61] Sukhotina IA, Malyshkin AA, Markou A, Bespalov AY. Lack of depression-like effects of saccharin deprivation in rats: forced swim test, differential reinforcement of low rates and intracranial selfstimulation procedures. Behav Neurosci 2003; 117(5): 970-7.

[62] Heath TP, Melichar JK, Nutt DJ, Donaldson LF. Human taste thresholds are modulated by serotonin and noradrenaline. J Neurosci 2006; 26(49): 12664-71.

[63] Pause BM, Miranda A, Goder R, Aldenhoff JB, Ferstl R. Reduced olfactory performance in patients with major depression. J Psychiatr Res 2001; 35(5): 271-7.

[64] Scinska A, Sienkiewicz-Jarosz H, Kuran W, et al. Depressive symptoms and taste reactivity in humans. Physiol Behav 2004; 82(5): 899-904.

[65] Steiner JE, Lidar-Lifschitz D, Perl E. Taste and odor: reactivity in depressive disorders, a multidisciplinary approach. Percept Mot Skills 1993;77(3 Pt 2.): 1331-46.

[66] Thomas HJ, Fries W, Distel H. Evaluation of olfactory stimuli by depressed patients. Nervenarzt 2002; 73(1): 71-7.

[67] Murison R, Hansen AL. Reliability of the chronic mild stress paradigm: implications for research and animal welfare. Integr Physiol Behav Sci 2001; 36: 266-74.

[68] Heckmann JG, Lang CJ. Neurological causes of taste disorders. Adv Otorhinolaryngol 2006; 63: 255-64

[69] Eibenstein A, Fioretti AB, Simaskou MN, et al. Olfactory screening test in mild cognitive impairment. Neurol Sci 2005; 26(3): 156-60.

[70] Zivadinov R, Zorzon M, Monti Bragadin L, Pagliaro G, Cazzato G. Olfactory loss in multiple sclerosis. J Neurol Sci 1999; 168(2): 127-30.

[71] Kudryavtseva NN, Amstislavskaya TG, Kucheryavy S. Effects of repeated aggressive encounters on approach to a female and plasma testosterone on male mice. Horm Behav 2004; 45(2): 103-7.

[72] Caramaschi D, de Boer SF, de Vries H, Koolhaas JM. Development of violence in mice through repeated victory along with changes in prefrontal cortex neurochemistry. Behav Brain Res 2008; 189(2): 263-72.

Received: December 30, 2008

Revised: March 27, 2009

Accepted: April 8, 2009

(c) Bottoni et al.; Licensee Bentham Open.

This is an open access article licensed under the terms of the Creative Commons Attribution Non-Commercial License (http://creativecommons.org/licenses/by$\mathrm{nc} / 3.0 /$ ) which permits unrestricted, non-commercial use, distribution and reproduction in any medium, provided the work is properly cited. 